\title{
CONSTANT MEAN CURVATURE HYPERSURFACES WITH CONSTANT $\delta$-INVARIANT
}

\author{
BANG-YEN CHEN and OSCAR J. GARAY
}

\author{
Received 29 April 2003
}

\begin{abstract}
We completely classify constant mean curvature hypersurfaces (CMC) with constant $\delta$-invariant in the unit 4-sphere $S^{4}$ and in the Euclidean 4-space $\mathbb{E}^{4}$.

2000 Mathematics Subject Classification: 53C40, 53B25, 53C42.
\end{abstract}

1. Introduction. A hypersurface in the unit round sphere $S^{n+1}$ is called isoparametric if it has constant principal curvatures. It is known from [1] that an isoparametric hypersurface in $S^{4}$ is either an open portion of a 3-sphere or an open portion of the product of a circle and a 2-sphere, or an open portion of a tube of constant radius over the Veronese embedding. Because every isoparametric hypersurface in $S^{4}$ has constant mean curvature (CMC) and constant scalar curvature, it is interesting to determine all hypersurfaces with CMC and constant scalar curvature. In [2], it was proved that a closed hypersurface with CMC and constant scalar curvature in $S^{4}$ is isoparametric. Furthermore, complete hypersurfaces with CMC and constant scalar curvature in $S^{4}$ or in $\mathbb{E}^{4}$ have been completely classified in [9].

For each Riemannian $n$-manifold $M^{n}$ with $n \geq 3$, the first author defined in $[3,4]$ the Riemannian invariant $\delta$ on $M$ by

$$
\delta(p)=\tau(p)-\inf K(p)
$$

where $\tau=\sum_{i<j} K\left(e_{i} \wedge e_{j}\right)$ is the scalar curvature and $\inf K$ is the function assigning to each $p \in M^{n}$ the infimum of $K(\pi), \pi$ running over all planes in $T_{p} M$. Although the invariant $\delta$ and the scalar curvature are both Riemannian scalar invariants, they are very much different in nature.

It is known that the invariant $\delta$ plays some important roles in recent study of Riemannian manifolds and Riemannian submanifolds (see, e.g., [4, 5, 6, 7, 8, 10 , $11,12,14,15,16])$. In particular, it was proved in [3] that for any submanifold of a real space form $R^{m}(\epsilon)$ of constant curvature $\epsilon$, one has the following general sharp inequality:

$$
\delta \leq \frac{n^{2}(n-2)}{2(n-1)} H^{2}+\frac{1}{2}(n+1)(n-2) \epsilon,
$$


where $H^{2}$ is the squared mean curvature function and $n$ is the dimension of the submanifold.

Clearly, every isoparametric hypersurface in $S^{4}$ or in $\mathbb{E}^{4}$ has constant mean curvature and constant $\delta$-invariant. So, it is a natural problem to study hypersurfaces in $S^{4}$ and $\mathbb{E}^{4}$ with CMC and constant $\delta$-invariant. The purpose of this paper is thus to classify such hypersurfaces.

Our main results are the following theorems.

THEOREM 1.1. A CMC hypersurface in the Euclidean 4-space $\mathbb{E}^{4}$ has constant $\delta$-invariant if and only if it is one of the following:

(1) an isoparametric hypersurface;

(2) a minimal hypersurface with relative nullity greater than or equal to 1 ;

(3) an open portion of a hypercylinder $N \times \mathbb{R}$ over a surface $N$ in $\mathbb{E}^{3}$ with CMC and nonpositive Gauss curvature.

THEOREM 1.2. A CMC hypersurface $M$ in the unit 4-sphere $S^{4}$ has constant $\delta$-invariant if and only if one of the following two statements holds:

(1) $M$ is an isoparametric hypersurface;

(2) there is an open dense subset $U$ of $M$ and a nontotally geodesic isometric minimal immersion $\phi: B^{2} \rightarrow S^{4}$ from a surface $B^{2}$ into $S^{4}$ such that $U$ is an open subset of $N B^{2} \subset S^{4}$, where $N B^{2}$ is defined by

$$
N_{p} B^{2}=\left\{\xi \in T_{\phi(p)} S^{4}:\langle\xi, \xi\rangle=1,\left\langle\xi, \phi_{*}\left(T_{p} B^{2}\right)\right\rangle=0\right\}
$$

In contrast to [2, 9], we do not make any global assumption on the hypersurfaces in Theorems 1.1 and 1.2.

As an immediate application of Theorem 1.1, we have the following corollary.

COROLLARY 1.3. Let $M$ be a complete hypersurface of Euclidean 4-space $\mathbb{E}^{4}$. Then $M$ has constant $\delta$-invariant and nonzero CMC if and only if $M$ is one of the following hypersurfaces:

(1) an ordinary hypersphere;

(2) a spherical hypercylinder: $\mathbb{R} \times S^{2}$;

(3) a hypercylinder over a circle: $\mathbb{E}^{2} \times S^{1}$.

2. Preliminaries. Let $R^{m}(4)$ denote the complete simply connected space form $R^{4}(\epsilon)$ of constant curvature $\epsilon$. Let $M$ be a hypersurface of an $R^{4}(\epsilon)$. Denote by $\nabla$ and $\tilde{\nabla}$ the Levi-Civita connections of $M^{n}$ and $R^{4}(\epsilon)$, respectively. Then the Gauss and Weingarten formulas of $M^{n}$ in $R^{4}(\epsilon)$ are given, respectively, by

$$
\tilde{\nabla}_{X} Y=\nabla_{X} Y+h(X, Y), \quad \tilde{\nabla}_{X} \xi=-A X
$$


for tangent vector fields $X, Y$, and unit normal vector field $\xi$, where $h$ denotes the second fundamental form and $A$ the shape operator. The second fundamental form and the shape operator are related by

$$
\langle A X, Y\rangle=\langle h(X, Y), \xi\rangle \text {. }
$$

The mean curvature $H$ of $M$ in $R^{4}(\epsilon)$ is defined by $H=(1 / 3) \operatorname{trace} A$. A hypersurface is called a CMC hypersurface if it has CMC.

Denote by $R$ the Riemann curvature tensor of $M$. Then the equation of Gauss is given by

$$
\begin{aligned}
R(X, Y ; Z, W)= & (\langle X, W\rangle\langle Y, Z\rangle-\langle X, Z\rangle\langle Y, W\rangle) \epsilon+\langle h(X, W), h(Y, Z)\rangle \\
& -\langle h(X, Z), h(Y, W)\rangle
\end{aligned}
$$

for vectors $X, Y, Z$, and $W$ tangent to $M$. The Codazzi equation is given by

$$
\left(\nabla_{X} A\right) Y=\left(\nabla_{Y} A\right)(X) .
$$

Since $A$ is a symmetric endomorphism of $T_{p} M, p \in M$, we have three eigenvalues $a, b$, and $c$ with three independent unit eigenvectors $e_{1}, e_{2}$, and $e_{3}$ so that

$$
A e_{1}=a e_{1}, \quad A e_{2}=b e_{2}, \quad A e_{3}=c e_{3},
$$

where $A=A_{e_{4}}$. The functions $a, b$, and $c$ are called the principal curvatures and $e_{1}, e_{2}$, and $e_{3}$ the principal directions.

With respect to the frame fields $e_{1}, e_{2}$, and $e_{3}$ of $M$ chosen above, let $\omega^{1}, \omega^{2}$, and $\omega^{3}$ be the field of dual frames and let $\omega_{B}^{A}, A, b=1,2,3,4$, be the connection forms associated with $e_{1}, e_{2}, e_{3}$, and $e_{4}$. Then the structure equations of $M$ in $R^{4}(\epsilon)$ are given by

$$
\begin{aligned}
& d \omega^{i}=-\sum_{j=1}^{3} \omega_{j}^{i} \wedge \omega^{j}, \quad \omega_{j}^{i}+\omega_{i}^{j}=0, \\
& d \omega_{j}^{i}=\sum_{k=1}^{3} \omega_{k}^{i} \wedge \omega_{k}^{j}+\omega_{i}^{4} \wedge \omega_{j}^{4}+\epsilon \omega^{i} \wedge \omega^{j}, \\
& d \omega_{i}^{4}=\sum_{j=1}^{3} \omega_{j}^{4} \wedge \omega_{j}^{i}, \quad i, j=1,2,3 .
\end{aligned}
$$

Moreover, from (2.5), we have

$$
\omega_{1}^{4}=a \omega^{1}, \quad \omega_{2}^{4}=b \omega^{2}, \quad \omega_{3}^{4}=c \omega^{3} .
$$

Without loss of generality, we may choose $e_{1}, e_{2}$, and $e_{3}$ such that $a \geq b \geq c$. It is well known that $a, b$, and $c$ are continuous on $M$ and differentiable on the 
open subset $U=\{p \in M: a(p)>b(p)>c(p)\}$. The principal directions $e_{1}, e_{2}$, and $e_{3}$ can be chosen to be differentiable on $U$.

Let $p$ be any given point in $M$. If $0>b \geq c$ at $p$, then, after replacing $\xi$ by $-\xi$ and interchanging $a$ and $c$, we obtain $a \geq b>0$ and $b \geq c$.

3. Lemmas. We follow the notations given in Section 2. Throughout this paper, we will choose $e_{1}, e_{2}, e_{3}$, and $e_{4}$ so that $a \geq b \geq 0$ and $b \geq c$.

LEMMA 3.1. For each point $p \in M$, either

(a) $\inf K=b c+\epsilon$ with $c \geq 0$ at $p$, or

(b) $\inf K=a c+\epsilon$ with $c \leq 0$ at $p$.

Proof. Recall that we have assumed that $a \geq b \geq 0$ and $b \geq c$ at $p$. Let $P$ be any 2-plane in $T_{p} M$. Then $P$ must intersects the 2-plane $\operatorname{Span}\left\{e_{1}, e_{2}\right\}$. Thus, there exists an orthonormal basis $\{X, Y\}$ of $P$ such that $X \in P \cap \operatorname{Span}\left\{e_{1}, e_{2}\right\}$ and

$$
\begin{gathered}
X=\cos \theta e_{1}+\sin \theta e_{2}, \\
Y= \pm \sin \theta \cos \phi e_{1} \mp \cos \theta \cos \phi e_{2}+\sin \phi e_{3}
\end{gathered}
$$

for some $\theta$ and $\phi$ with $\theta \in[0, \pi), \phi \in[0, \pi]$. It is easy to see that the sectional curvature $K(P)$ of $P$ is given by

$$
K(P)=a b \cos ^{2} \phi+c\left(a \cos ^{2} \theta+b \sin ^{2} \theta\right) \sin ^{2} \phi+\epsilon .
$$

We regard the sectional curvature at $p$ as a function $K(\theta, \phi)$ of $\theta$ and $\phi$.

If $c \geq 0$, (3.2) can be expressed as

$$
K(\theta, \phi)=a c+a(b-c) \cos ^{2} \phi-c(a-b) \sin ^{2} \theta \sin ^{2} \phi+\epsilon,
$$

which implies that $K(\theta, \phi) \geq b c+\epsilon$ with the equality holding at $(\theta, \phi)=$ $(\pi / 2, \pi / 2)$.

If $c \leq 0$, we can express (3.2) as

$$
K(\theta, \phi)=b c+b(a-c) \cos ^{2} \phi+c(a-b) \cos ^{2} \theta \sin ^{2} \phi+\epsilon
$$

which implies that $K(\theta, \phi) \geq a c+\epsilon$ with the equality holding at $(\theta, \phi)=$ $(0, \pi / 2)$.

LEMMA 3.2. On the open subset $U$ on which $M$ has three distinct principal curvatures, the following equations hold: 


$$
\begin{gathered}
e_{2} a=(a-b) \omega_{1}^{2}\left(e_{1}\right), \\
e_{3} a=(a-c) \omega_{1}^{3}\left(e_{1}\right), \\
e_{3} b=(b-c) \omega_{2}^{3}\left(e_{2}\right), \\
e_{1} b=(b-a) \omega_{2}^{1}\left(e_{2}\right), \\
e_{1} c=(c-a) \omega_{3}^{1}\left(e_{3}\right), \\
e_{2} c=(c-b) \omega_{3}^{2}\left(e_{3}\right), \\
(c-b) \omega_{3}^{2}\left(e_{1}\right)=(c-a) \omega_{3}^{1}\left(e_{2}\right), \\
(b-c) \omega_{2}^{3}\left(e_{1}\right)=(b-a) \omega_{2}^{1}\left(e_{3}\right), \\
(a-b) \omega_{1}^{2}\left(e_{3}\right)=(a-c) \omega_{1}^{3}\left(e_{2}\right) .
\end{gathered}
$$

Proof. The proof follows from Codazzi's equation and is a straightforward computation.

4. Proofs of Theorems 1.1 and 1.2. We use the same notations as before. Let $M$ be a (connected) CMC hypersurface with constant $\delta$-invariant in $R^{4}(\epsilon)$. Then the scalar curvature $\tau$ of $M$ is given by

$$
\tau=a b+b c+a c+3 \epsilon .
$$

From the constancy of the mean curvature, we have

$$
a+b+c=r_{1}
$$

for some constant $r_{1}$. By combining Lemma 3.1 with (1.1) and (4.1), we obtain

(i) $\delta=a(b+c)+2 \epsilon$ with $c \geq 0$, or

(ii) $\delta=b(a+c)+2 \epsilon$ with $c \leq 0$.

When $U=\{p \in M: a(p)>b(p)>c(p)\}$ is empty, $M$ is an isoparametric hypersurface since the mean curvature and the $\delta$-invariant are both constant. Thus, from now on, we may assume that $U$ is nonempty and work on $U$.

We will treat Cases (i) and (ii) on $U$ separately.

CASE (i) $(\delta=a(b+c)+2 \epsilon, c \geq 0)$. Since $\delta$ is constant, we get $a(b+c)=$ $r_{2}-2 \epsilon$ for some constant $r_{2}$. Combining this with (4.2) yields

$$
a=c_{1}, \quad b+c=c_{2}
$$

for some constants $c_{1}$ and $c_{2}$. For simplicity, let

$$
\omega_{3}^{2}\left(e_{1}\right)=\mu, \quad \omega_{1}^{2}\left(e_{2}\right)=f, \quad \omega_{2}^{3}\left(e_{2}\right)=g, \quad \omega_{2}^{3}\left(e_{3}\right)=h .
$$

If $b$ and $c$ are constant, then $M$ is isoparametric. So, we assume that $b$ and $c$ are nonconstant on $U$. Using (4.3), we get $e_{j} b=-e_{j} c, j=1,2,3$. Thus, 
Lemma 3.2 gives

$$
\begin{gathered}
\omega_{1}^{3}\left(e_{1}\right)=\omega_{1}^{2}\left(e_{1}\right)=0, \\
e_{1} b=(a-b) f=(c-a) \omega_{1}^{3}\left(e_{3}\right), \\
e_{2} b=(b-c) h, \\
e_{3} b=(b-c) g .
\end{gathered}
$$

From (4.5), we know that the integral curves of $e_{1}$ are geodesics in $U$. Applying (3.12), (3.13), (4.6), (4.7), and (4.8), we find

$$
\begin{gathered}
e_{1} b=(a-b) f, \quad e_{2} b=(c-b) h, \\
e_{3} b=(b-c) g, \quad e_{j} a=0, \quad e_{j} c=-e_{j} b, \\
\omega_{1}^{2}=f \omega^{2}+\frac{b-c}{b-a} \mu \omega^{3}, \\
\omega_{1}^{3}=\frac{b-c}{c-a} \mu \omega^{2}+\frac{a-b}{c-a} f \omega^{3}, \\
\omega_{2}^{3}=-\mu \omega^{1}+g \omega^{2}+h \omega^{3},
\end{gathered}
$$

for $j=1,2,3$. By applying (2.6), (4.9), (4.10), (4.11), and (4.12), we find

$$
\begin{gathered}
d \omega^{1}=\left(\frac{b-c}{a-b}+\frac{b-c}{c-a}\right) \mu \omega^{2} \wedge \omega^{3}, \\
d \omega^{2}=f \omega^{1} \wedge \omega^{2}+\frac{a-c}{b-a} \mu \omega^{1} \wedge \omega^{3}+g \omega^{2} \wedge \omega^{3}, \\
d \omega^{3}=\frac{a-b}{a-c} \mu \omega^{1} \wedge \omega^{2}-\frac{a-b}{a-c} f \omega^{1} \wedge \omega^{3}+h \omega^{2} \wedge \omega^{3} .
\end{gathered}
$$

Using $\left(\nabla_{e_{2}} e_{1}-\nabla_{e_{1}} e_{2}-\left[e_{2}, e_{1}\right]\right) b=0$, we get

$$
(a-b) e_{2} f+(b-c) e_{1} h=2(c-a) f h+\frac{(b-a)(b-c)}{c-a} \mu g .
$$

Similarly, from $\left(\nabla_{e_{3}} e_{1}-\nabla_{e_{1}} e_{3}-\left[e_{3}, e_{1}\right]\right) b=\left(\nabla_{e_{3}} e_{2}-\nabla_{e_{2}} e_{3}-\left[e_{3}, e_{2}\right]\right) b=0$, we get

$$
\begin{aligned}
(a-b) e_{3} f+(c-b) e_{1} g= & \frac{(a-c)(c-b)}{b-a} \mu h+\frac{2 a(b+c)-b^{2}-c^{2}-2 a^{2}}{c-a} f g, \\
& e_{3} h+e_{2} g=\frac{c-b}{a-c} \mu f .
\end{aligned}
$$


By computing $d \omega_{1}^{2}$ and applying (4.10), (4.11), (4.12), (4.13), and Cartan's structure equations, we obtain

$$
\begin{gathered}
e_{1} f=\frac{2(b-c)}{a-c} \mu^{2}-f^{2}-a b-\epsilon, \\
e_{1}\left(\frac{b-c}{b-a} \mu\right)=\left\{\frac{b-c}{a-b}+\frac{2(a-b)}{a-c}\right\} \mu f, \\
e_{3} f+e_{2}\left(\frac{b-c}{a-b} \mu\right)=\frac{b+c-2 a}{c-a}\left\{f g-\frac{b-c}{a-b} \mu h\right\} .
\end{gathered}
$$

Similarly, by computing $d \omega_{1}^{3}$ and $d \omega_{2}^{3}$, and by applying (4.10), (4.11), (4.12), (4.13), and Cartan's structure equations, we obtain

$$
\begin{gathered}
e_{1}\left(\frac{b-c}{c-a} \mu\right)=\left\{\frac{2 a^{2}+2 c^{2}+b^{2}-a b-3 a c-b c}{(a-c)^{2}}\right\} \mu f, \\
e_{1}\left(\frac{a-b}{c-a} f\right)=-a c-\epsilon-\left(\frac{a-b}{c-a}\right)^{2} f^{2}+\frac{2(b-c)}{b-a} \mu^{2}, \\
e_{3}\left(\frac{c-b}{c-a} \mu\right)+e_{2}\left(\frac{a-b}{c-a} f\right)=\frac{2 a-b-c}{a-c}\left\{f h+\frac{b-c}{a-b} \mu g\right\}, \\
e_{2} \mu+e_{1} g=\frac{a-b}{c-a} \mu h-f g, \\
e_{1} h+e_{3} \mu=\frac{a-b}{a-c} f h+\frac{a-c}{a-b} \mu g, \\
e_{2} h-e_{3} g=\frac{2(b-c)^{2} \mu^{2}}{(a-b)(a-c)}+\frac{a-b}{a-c} f^{2}-g^{2}-h^{2}-b c-\epsilon .
\end{gathered}
$$

Combining (4.9), (4.16), and (4.20) yields

$$
\begin{aligned}
& 2(2 a-b-c)(a-b)^{2}+2(2 a-b-c)(b-c)^{2} \mu^{2} \\
& \quad+(a-b)(a-c)\{a b(a-b)+a c(a-c)+(2 a-b-c) \epsilon\}=0,
\end{aligned}
$$

which is impossible unless $\epsilon<0$, since we assume that $a>b>c \geq 0$ in Case (i).

CASE (ii) $(\delta=b(a+c)+2 \epsilon, c \leq 0)$. Since $\delta$ is constant, we get $b(a+c)=$ $r_{2}-2 \epsilon$ for some constant $r_{2}$. Combining this with (4.2) yields

$$
b=c_{3}, \quad a+c=c_{4},
$$

for some constants $c_{3}$ and $c_{4}$. For simplicity, let

$$
\omega_{3}^{1}\left(e_{2}\right)=\tilde{\mu}, \quad \omega_{2}^{1}\left(e_{1}\right)=\tilde{f}, \quad \omega_{1}^{3}\left(e_{1}\right)=\tilde{g}, \quad \omega_{1}^{3}\left(e_{3}\right)=\tilde{h} .
$$

If $a$ and $c$ are constant, then $M$ is isoparametric. So, from now on, we may assume that $a$ and $c$ are nonconstant on $U$. Using (4.26), we get

$$
e_{j} a=-e_{j} c, \quad j=1,2,3 .
$$


Thus, Lemma 3.2 yields

$$
\begin{aligned}
\omega_{2}^{3}\left(e_{2}\right) & =\omega_{2}^{1}\left(e_{2}\right)=0, \\
e_{1} a=(c-a) \tilde{h}, \quad e_{2} a & =(b-a) \tilde{f}, \quad e_{3} a=(a-c) \tilde{g} .
\end{aligned}
$$

Equation (4.28) shows that the integral curves of $e_{2}$ are geodesics in $U$. Applying (3.12), (3.13), (4.29), and (4.30), we find

$$
\begin{aligned}
& \omega_{1}^{2}=-\tilde{f} \omega^{1}-\frac{a-c}{a-b} \tilde{\mu} \omega^{3}, \\
& \omega_{1}^{3}=\tilde{g} \omega^{1}-\tilde{\mu} \omega^{2}+\tilde{h} \omega^{3}, \\
& \omega_{2}^{3}=\frac{a-c}{c-b} \tilde{\mu} \omega^{1}+\frac{a-b}{b-c} \tilde{f} \omega^{3} .
\end{aligned}
$$

By applying (2.6), (4.31), (4.32), and (4.33), we find

$$
\begin{gathered}
d \omega^{1}=-\tilde{f} \omega^{1} \wedge \omega^{2}+\tilde{g} \omega^{1} \wedge \omega^{3}+\frac{b-c}{a-b} \tilde{\mu} \omega^{2} \wedge \omega^{3}, \\
d \omega^{2}=-\left(\frac{a-c}{a-b}+\frac{a-c}{b-c}\right) \tilde{\mu} \omega^{1} \wedge \omega^{3}, \\
d \omega^{3}=\frac{a-b}{b-c} \tilde{\mu} \omega^{1} \wedge \omega^{2}+\tilde{h} \omega^{1} \wedge \omega^{3}+\frac{a-b}{b-c} \tilde{f} \omega^{2} \wedge \omega^{3} .
\end{gathered}
$$

Using $\left(\nabla_{e_{2}} e_{1}-\nabla_{e_{1}} e_{2}-\left[e_{2}, e_{1}\right]\right) a=0$, we find

$$
(a-b) e_{1} \tilde{f}-(a-c) e_{2} \tilde{h}=2(b-a) \tilde{f} \tilde{h}+\frac{(a-b)(a-c)}{b-c} \tilde{\mu} \tilde{g} .
$$

Similarly, from $\left(\nabla_{e_{3}} e_{1}-\nabla_{e_{1}} e_{3}-\left[e_{3}, e_{1}\right]\right) a=\left(\nabla_{e_{3}} e_{2}-\nabla_{e_{2}} e_{3}-\left[e_{3}, e_{2}\right]\right) a=0$, we get

$$
\begin{gathered}
(a-b) e_{3} \tilde{f}+(a-c) e_{2} \tilde{g}=\frac{(a-c)(b-c)}{a-b} \tilde{\mu} \tilde{h}+\frac{2 a b+2 b c-2 b^{2}-a^{2}-c^{2}}{b-c} \tilde{f} \tilde{g}, \\
e_{3} \tilde{h}+e_{1} \tilde{\mathfrak{g}}=\frac{c-a}{b-c} \tilde{\mu} \tilde{f} .
\end{gathered}
$$

By computing $d \omega_{1}^{2}$ and applying (4.31), (4.32), and (4.33) and Cartan's structure equations, we obtain

$$
\begin{gathered}
e_{2} \tilde{f}=\frac{2(a-c)}{b-c} \tilde{\mu}^{2}-\tilde{f}^{2}-a b-\epsilon, \\
e_{2}\left(\frac{a-c}{a-b} \tilde{\mu}\right)=\left\{\frac{a-c}{b-a}-\frac{2(a-b)}{b-c}\right\} \tilde{\mu} \tilde{f}, \\
e_{3} \tilde{f}+e_{1}\left(\frac{a-c}{b-a} \tilde{\mu}\right)=\frac{a+c-2 b}{c-b}\left\{\tilde{f} \tilde{g}+\frac{a-c}{a-b} \tilde{\mu} \tilde{h}\right\} .
\end{gathered}
$$


Similarly, by computing $d \omega_{1}^{3}, d \omega_{2}^{3}$, and by applying (4.31), (4.32), and (4.33) and Cartan's structure equations, we obtain

$$
\begin{gathered}
e_{2}\left(\frac{a-c}{c-b} \tilde{\mu}\right)=\left\{\frac{2 b^{2}+2 c^{2}+a^{2}-a b-3 b c-a c}{(b-c)^{2}}\right\} \tilde{\mu} \tilde{f}, \\
e_{2}\left(\frac{a-b}{b-c} \tilde{f}\right)=-b c-\epsilon-\left(\frac{a-b}{c-b}\right)^{2} \tilde{f}^{2}+\frac{2(a-c)}{a-b} \tilde{\mu}^{2}, \\
e_{3}\left(\frac{a-c}{b-c} \tilde{\mu}\right)+e_{1}\left(\frac{a-b}{b-c} \tilde{f}\right)=\frac{2 b-a-c}{b-c}\left\{\tilde{f} \tilde{h}-\frac{a-c}{a-b} \tilde{\mu} \tilde{g}\right\}, \\
e_{1} \tilde{\mu}+e_{2} \tilde{g}=\frac{a-b}{b-c} \tilde{\mu} \tilde{h}-\tilde{f} \tilde{g}, \\
e_{2} \tilde{h}+e_{3} \tilde{\mu}=\frac{b-a}{b-c} \tilde{f} \tilde{h}-\frac{b-c}{a-b} \tilde{\mu} \tilde{g}, \\
e_{1} \tilde{h}-e_{3} \tilde{g}=\frac{2(a-c)^{2} \tilde{\mu}^{2}}{(b-a)(b-c)}-\frac{a-b}{b-c} \tilde{f}^{2}-\tilde{g}^{2}-\tilde{h}^{2}-a c-\epsilon .
\end{gathered}
$$

Applying (4.30), (4.37), and (4.41) yields

$$
\begin{aligned}
2(2 b-a-c)(a-b)^{2} \tilde{f}^{2}+2(2 b-a-c)(a-c)^{2} \tilde{\mu}^{2} \\
\quad+(b-a)(b-c)\{a b(b-a)+b c(b-c)+(2 b-a-c) \epsilon\}=0 .
\end{aligned}
$$

Using (4.26), (4.30), and (4.38), we find

$$
e_{2} \tilde{\mu}=\frac{2\left[(a-b)^{2}+(b-c)^{2}\right]}{(a-c)(c-b)} \tilde{\mu} \tilde{f} .
$$

On the other hand, by differentiating (4.46) with respect to $e_{2}$ and using (4.26), (4.30), and (4.37), we obtain

$$
\begin{aligned}
4(a+c-2 b)(a-c)^{2} \tilde{\mu}\left(e_{2} \tilde{\mu}\right) & \\
= & b(a-b)\left(3 a^{3}-13 a^{2} b+10 a b^{2}+7 a^{2} c-4 a b c-2 b^{2} c-3 a c^{2}+b c^{2}+c^{3}\right) \tilde{f} \\
& -8 \frac{(a+c-2 b)^{2}(a-b)(a-c)}{b-c} \tilde{\mu}^{2} \tilde{f}+8(a+c-2 b)(a-b)^{2} \tilde{f}^{3} \\
& -(a-b)(a+c-2 b)(4 b-3 a-c) \epsilon \tilde{f} .
\end{aligned}
$$

Replacing $\tilde{f}^{2}$ in (4.48) by using (4.46) yields

$$
\begin{aligned}
4(a+c-2 b)(a-c)^{2} \tilde{\mu}\left(e_{2} \tilde{\mu}\right) \\
=3(a-b)(a-c)(a+c-2 b) \tilde{\epsilon} \tilde{f} \\
\quad+3 b(a-b)(a-c)\left(a^{2}-3 a b+2 b^{2}+2 a c-3 b c+c^{2}\right) \tilde{f} \\
\quad-8 \frac{(a+c-2 b)(a-c)\left[(a-b)^{2}+(b-c)^{2}\right]}{b-c} \tilde{\mu}^{2} \tilde{f} .
\end{aligned}
$$


Substituting (4.47) into (4.49) yields

$$
\tilde{f}(a+c-2 b)\{b(a+c-b)+\epsilon\}=0 .
$$

CASE (ii-a) $(\tilde{f}=0)$. In this case, (4.37) and (4.41) imply that

$$
2(a-c) \mu^{2}=(a b+\epsilon)(b-c)=(b c+\epsilon)(a-b) .
$$

The equality in (4.51) yields

$$
b(a b+b c-2 a c)=(a+c-2 b) \epsilon .
$$

CASE (ii-a.1) $(\tilde{f}=0, b \neq 0)$. In this case, (4.26) and (4.52) imply that $a c$ is constant. Hence, by (4.26), we know that both $a$ and $c$ are constant. Thus, $M$ is isoparametric.

CASE (ii-a.2) ( $b=\tilde{f}=0, \epsilon=1)$. In this case, (4.52) reduces to $a+c=2 b$. So, $M$ satisfies the equality case of inequality (1.2). Therefore, by applying [7, Theorem 2], we know that $M$ is given by Theorem 1.2 (2).

CASE (ii-a.3) $(b=\tilde{f}=\epsilon=0)$. In this case, (4.37) implies that $\tilde{\mu}=0$. Thus, by (4.31) and (4.33), we obtain $\omega_{1}^{2}=\omega_{2}^{3}=0$. On the other hand, from (4.29), we have $\nabla_{e_{2}} e_{2}=0$. Therefore, $\mathscr{D}_{1}=\operatorname{Span}\left\{e_{1}, e_{3}\right\}$ and $\mathscr{D}_{2}=\operatorname{Span}\left\{e_{2}\right\}$ are integrable distributions in $M$ with totally geodesic leaves. Hence, $M$ is locally the Riemannian product of a line and a Riemannian 2-manifold $N^{2}$. Moreover, because the second fundamental form $h$ of $M$ in $\mathbb{E}^{4}$ satisfies $h\left(\mathscr{D}_{1}, \mathscr{D}_{2}\right)=\{0\}$, Moore's lemma [13] implies that $M$ is an open portion of a hypercylindrical $\mathbb{R} \times N^{2} \subset \mathbb{E} \times \mathbb{E}^{3}=\mathbb{E}^{4}$. Furthermore, from the assumption on the shape operator of $M$ in $\mathbb{E}^{4}$, we know that the mean curvature of $N$ in $\mathbb{E}^{3}$ is constant and the Gauss curvature of $N$ is nonpositive. Thus, we obtain case (3) of Theorem 1.1.

CASE (ii-b) $(\tilde{f} \neq 0, b=0)$. In this case, (4.50) yields $(a+c) \epsilon=0$.

If $\epsilon=1$, then $a+c=0$. Hence, $M$ is a minimal hypersurface satisfying the equality case of inequality (1.2). Thus, by applying [7, Theorem 2], we obtain case (2) of Theorem 1.2.

If $\epsilon=0$, then (4.46) implies that $a+c-2 b=0$ due to $b=0$ and $a \neq b$. Hence, $M$ satisfies the equality case of inequality (1.2). Since $M$ has CMC, [7, Theorem 1] implies that $M$ is either an isoparametric hypersurface or a minimal hypersurface which satisfies the equality $\delta=0$. Hence, we obtain either case (1) or case (2) of Theorem 1.1.

CASE (ii-c) $(b \neq 0, \tilde{f} \neq 0)$. In this case, (4.50) yields

$$
(a+c-2 b)\{b(a+c-b)+\epsilon\}=0 .
$$

If $a+c-2 b=0$ holds, then (4.46) implies that $a(a-b)-c(b-c)=0$ which is impossible, since $a \geq 0, c \leq 0$, and $a>b>0$ by assumption. Therefore, we 
must have

$$
\epsilon=b(b-a-c)
$$

From (4.54), $\epsilon \geq 0$, and $b>0$, we get

$$
b \geq a+c .
$$

On the other hand, by substituting (4.54) into (4.46), we find

$$
(a+c-2 b)\left[(a-c)^{2} \tilde{\mu}^{2}+(a-b)^{2} \tilde{f}^{2}\right]=b(b-a)^{2}(b-c)^{2} .
$$

In particular, we obtain $a+c>2 b$. Combining this with (4.55) gives $b<0$ which is a contradiction. Thus, this case is impossible.

The converse follows from [7, Theorem 2] and from direct computation.

ACKNOWLEDGMENT. The second author was supported by Grants no. 9/UPV00127.310-13574/01 and BFM2001-2871-C04-03.

\section{REFERENCES}

[1] E. Cartan, Sur des familles remarquables d'hypersurfaces isoparamétriques dans les espaces sphériques, Math. Z. 45 (1939), 335-367 (French).

[2] S. Chang, A closed hypersurface with constant scalar and mean curvatures in $S^{4}$ is isoparametric, Comm. Anal. Geom. 1 (1993), no. 1, 71-100.

[3] B.-Y. Chen, Some pinching and classification theorems for minimal submanifolds, Arch. Math. (Basel) 60 (1993), no. 6, 568-578.

[4] _ Strings of Riemannian invariants, inequalities, ideal immersions and their applications, The Third Pacific Rim Geometry Conference (Seoul, 1996), Monogr. Geom. Topology, vol. 25, Internat. Press, Massachusetts, 1998, pp. 7-60.

[5] _ Riemannian submanifolds, Handbook of Differential Geometry, Vol. I (F. Dillen and L. Verstraelen, eds.), North-Holland, Amsterdam, 2000, pp. 187-418.

[6] B.-Y. Chen and L. Vrancken, CR-submanifolds of complex hyperbolic spaces satisfying a basic equality, Israel J. Math. 110 (1999), 341-358.

[7] B.-Y. Chen and J. Yang, Elliptic functions, theta function and hypersurfaces satisfying a basic equality, Math. Proc. Cambridge Philos. Soc. 125 (1999), no. 3, 463-509.

[8] Corrigendum: "Elliptic functions, theta function and hypersurfaces satisfying a basic equality", Math. Proc. Cambridge Philos. Soc. 130 (2001), no. 1, 189-191.

[9] Q. M. Cheng and Q. R. Wan, Hypersurfaces of space forms $M^{4}(c)$ with constant mean curvature, Geometry and Global Analysis (Sendai, 1993), Tohoku Universty, Sendai, 1993, pp. 437-442.

[10] M. Dajczer and L. A. Florit, On Chen's basic equality, Illinois J. Math. 42 (1998), no. 1, 97-106.

[11] F. Dillen, M. Petrovic, and L. Verstraelen, Einstein, conformally flat and semisymmetric submanifolds satisfying Chen's equality, Israel J. Math. 100 (1997), 163-169. 
[12] F. Dillen and L. Vrancken, Totally real submanifolds in $S^{6}(1)$ satisfying Chen's equality, Trans. Amer. Math. Soc. 348 (1996), no. 4, 1633-1646.

[13] J. D. Moore, Isometric immersions of riemannian products, J. Differential Geometry 5 (1971), 159-168.

[14] T. Sasahara, Chen invariant of CR-submanifolds, Sūrikaisekikenkyūsho Kōkyūroku (2001), no. 1206, 114-120 (Japanese).

[15] _ On Chen invariant of CR-submanifolds in a complex hyperbolic space, Tsukuba J. Math. 26 (2002), no. 1, 119-132.

[16] B. Suceavă, The Chen invariants of warped products of hyperbolic planes and their applications to immersibility problems, Tsukuba J. Math. 25 (2001), no. 2, 311-320.

Bang-Yen Chen: Department of Mathematics, Michigan State University, East Lansing, MI 48824-1027, USA

E-mail address: bychen@math.msu.edu

Oscar J. Garay: Departamento de Matemáticas, Universidad del País Vasco/Euskal Herriko Unibertsitatea, Apartado 644. 48080 Bilbao, Spain

E-mail address: mtpgabeo@1g.ehu.es 


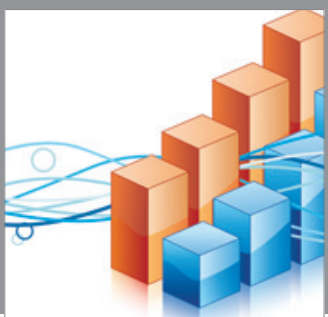

Advances in

Operations Research

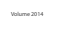

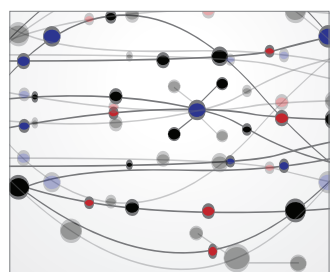

\section{The Scientific} World Journal
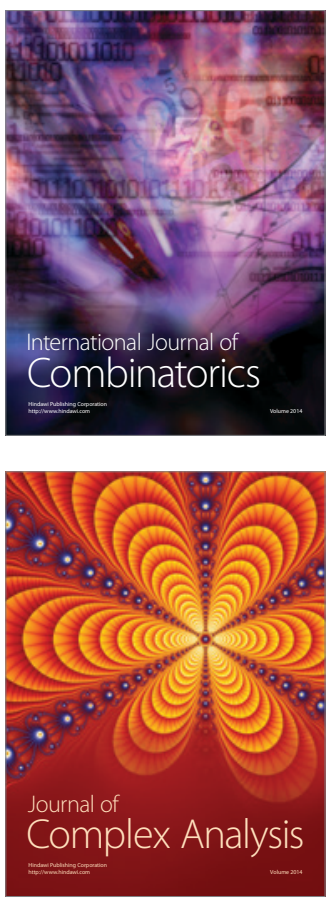

International Journal of

Mathematics and

Mathematical

Sciences
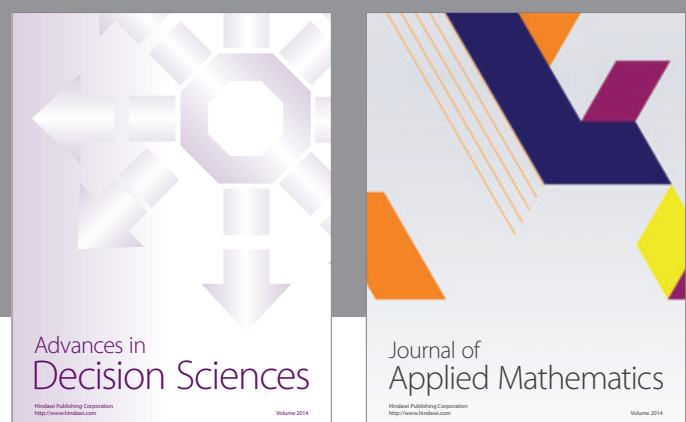

Journal of

Applied Mathematics
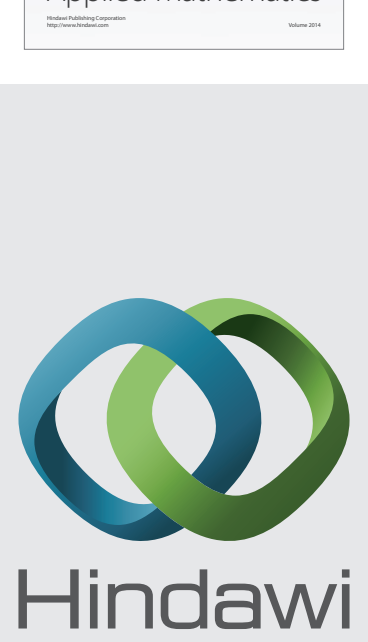

Submit your manuscripts at http://www.hindawi.com
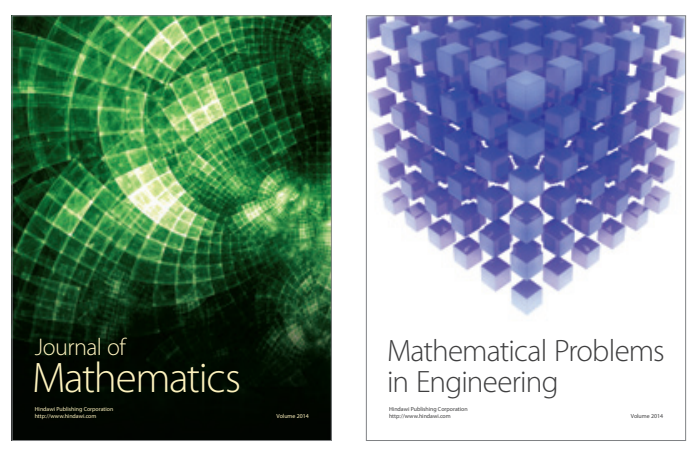

Mathematical Problems in Engineering
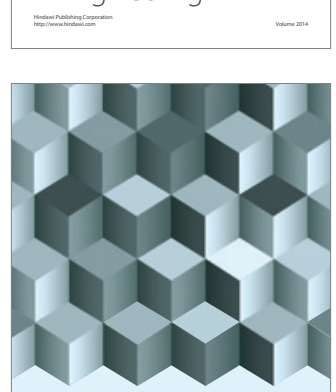

Journal of

Function Spaces
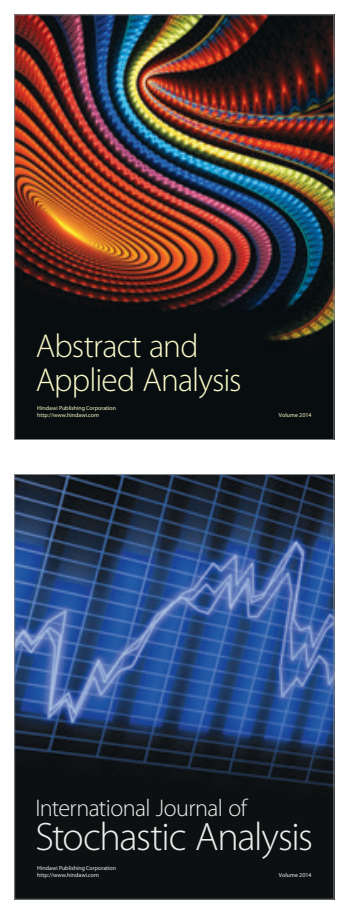

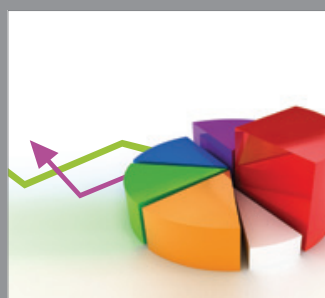

ournal of

Probability and Statistics

Promensencen
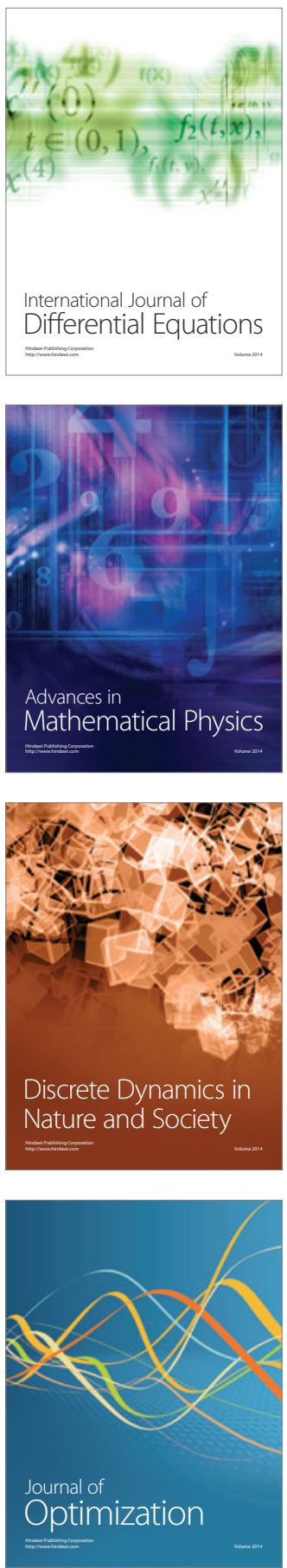